\title{
nrf2 Expressed by Bone
}

\author{
Eiichi Hinoi, Liyang Wang, Sayumi Fujimori and Yukio Yoneda*
}

\author{
Laboratory of Molecular Pharmacology, Division of Pharmaceutical Sciences, Kanazawa University Graduate School of Natural \\ Science and Technology, Kanazawa, Ishikawa 920-1192, Japan
}

\begin{abstract}
Nuclear factor E2-related factor 2 (nrf2) is accepted as an important transcription factor in the regulation of many phase II detoxifying enzymes and oxidative-stress-inducible genes in many tissues including bone and cartilage. However, little attention has been paid to precise role of nrf2 on osteoblasts and chondrocytes. In the present study, we therefore investigated the role of nrf2 in the regulation of cell proliferation, differentiation and maturation using both MC3T3-E1 and ATDC5 cells. In MC3T3-E1 cells stably transfected with nrf2 (MC3T3-E1-nrf2 clone), the differentiation-dependent induction of alkaline phosphatase activity was significantly inhibited in addition to decrease of the mineralized matrix formation. Moreover, expression of mRNAs for osteocalcin and type I collagen was also markedly attenuated in MC3T3E1-nrf2 clone but not in MC3T3E1-empty vector clone. These inhibitory effect on cell differentiation was also observed in ATDC5 cells stably transfected with nrf2. Moreover, nrf2 significantly impaired the runx2-dependent enhancement of osteocalcin promoter activity. These data suggest that nrf2 may negatively regulate the differentiation of both osteoblasts and chondrocytes through inhibition of the runx2 dependent transcriptional activity.
\end{abstract}

Key words: osteoblasts, chondrocytes, transcription factors, nrf2, runx2

\section{Introduction}

In bone tissues, two distinct cell types are known to sophisticatedly regulate bone formation and maintenance ${ }^{1,2)}$. These are bone-forming osteoblasts and bone-resorbing osteoclasts. The osteoblast lineage is derived from primitive multipotent mesenchymal stem cells with potentiality to differentiate into bone marrow stromal cells, chondrocytes, muscles and adipocytes ${ }^{3)}$, while osteoclasts are multinucleated cells derived from the fusion of mononuclear hematopoietic precursors ${ }^{4}$. The development and differentiation of these two distinct cells are under the tight regulation by a number of transcription factors. Among them, Runx2, a cell-specific member of the Runt family of transcription factors, play a pivotal role in osteoblastic differentiation. Runx2 is the earliest and most specific molecular marker of the osteoblast lineage, its expression is both necessary and sufficient to induce osteoblast differentiation, and it regulates expression of most genes characteristic of the osteoblast phenotype ${ }^{5,6,7)}$. Runx2 is also required for chondrocyte hypertrophy in bones ossifying through endochondral ossification ${ }^{8)}$.

Transcription factor nuclear factor E2-related factor 2 (nrf2) belongs to the cap-n-collar family of activators that shares a highly conserved basic region-leucine zipper structure ${ }^{9)}$. Nrf2 forms heterodimers with the small Maf proteins, and binds to the antioxidant responsive element or electrophile responsive elements of target genes, followed by regulation of many phase II detoxifying enzymes and oxidative-stress-inducible genes in many tissues including bone and cartilage ${ }^{10,11)}$ However, little attention has been paid to precise role of nrf2 on osteoblasts and chondrocytes. In the present study, we therefore investigated the role of nrf2 in the regulation of cell proliferation, differentiation and maturation using both MC3T3-E1 and ATDC5 cells.

\section{Materials and Methods}

Corespondence to Dr. Yukio Yoneda.,Laboratory of Molecular Pharmacology, Division of Pharmaceutical Sciences, Kanazawa University Graduate School of Natural Science and Technology, Kakuma-machi, Kanazawa, Ishikawa 920-1192, Japan, Tel/Fax: 81-(0)76-234-4471E-mail: yyoneda@anet.ne.jp
In situ hybridization analysis

Sections mounted were fixed with $4 \%$ paraformaldehyde, followed by treating with $0.2 \mathrm{M} \mathrm{HCl}$ and subsequent treating with $10 \mathrm{mg} / \mathrm{mL}$ Proteinase K. Sections were then subjected to acetylation in $0.1 \mathrm{M}$ triethanolamine $/ 0.25 \%$ acetic anhydride and subsequent stepwise dehydration in 70, 80, 90, 95 and $100 \%$ ethanol. After being dried, sections were covered with digoxigenin (DIG)-labeled cRNA probes at $65^{\circ} \mathrm{C}$ for $16 \mathrm{~h}$. Post-hybridization washes were done stepwise with 4 XSSC at $65^{\circ} \mathrm{C}, 50 \%$ formamide in $2 \mathrm{XSSC}$ at $65^{\circ} \mathrm{C}, 4 \mathrm{mg} / \mathrm{mL}$ RNase $\mathrm{A}$ at $37^{\circ} \mathrm{C}, 2 \mathrm{XSSC}$ at $65^{\circ} \mathrm{C}$, $0.2 \mathrm{XSSC}$ at $65^{\circ} \mathrm{C}$, and then $1.5 \%$ blocking buffer. Subsequently, sections were incubated with anti-DIG-AP antibody at $4^{\circ} \mathrm{C}$ for 16 h, followed by treating with NBT/BCIP for different periods.

\section{Stable transfection}

MC3T3-E1 and ATDC5 cells were plated at a density of $1.5 \mathrm{x}$ $10^{5}$ cells $/ \mathrm{cm}^{2}$. After $24 \mathrm{~h}$, they were stably transfected with $\mathrm{pEF}$ containing the full-length coding region of nrf2 (MC3T3-E1-nrf2 and ATDC5-nrf2) or with the empty vector (MC3T3-E1-EV and ATDC5-EV) using $2 \mu \mathrm{g}$ of DNA and Lipofectamine and Plus regent (Invitrogen, Carlsbad, CA) in $10 \mathrm{ml}$ of medium. After 24 $\mathrm{h}$, and every $48 \mathrm{~h}$ thereafter for 2 weeks, media were replaced with fresh media containing $600 \mu \mathrm{g} / \mathrm{ml}$ of G418. This dose and duration of treatment resulted in the death of all nontransfected cells within 10 days. Pools of 10 clones of MC3T3-E1-nrf2 and ATDC5-nrf2 were isolated for further studies. Pools of clones between passages 2 and 5 were used for the experiments reported.

\section{Constructs and Luciferase Assay}

A full-length mouse osteocalcin promoter with luciferase as the reporter gene (OG2) was generously gift from Dr. Karsenty. OG2 reporter was co-transfected with TK-Renilla luciferase construct in either the presence or absence of nrf2 and/or runx2 expression vector into MC3T3-E1 cells and COS7 cells. Two days after transfection, cells were lysed, and luciferase activity was determined using specific substrates in a luminometer according to the manufacture's protocol (Promega Madison, MI). Transfection efficiency was normalized by determining the activity 
of Renilla luciferase.

\section{Data analysis}

Results are all expressed as the mean \pm S.E. and the statistical significance was determined by the two-tailed and unpaired Students' $t$-test or the one-way analysis of variance ANOVA with Bonferroni/Dunnett post hoc test.

\section{Results \\ Expression of nrf2 in bone and cartilage}

In order to at first evaluate the possible expression of nrf2 in bone and cartilage, in situ hybridization was conducted on sections dissected from neonatal mouse tibia. type I collagen was specifically expressed by osteoblasts without any layers of differentiating chondrocytes. In addition, type II collagen was preferentially expressed by proliferating to prehypertrophic chondrocytes and type $\mathrm{X}$ collagen was highly expressed by hypertrophic chondrocytes. Under the experimental conditions used here, nrf2 was expressed in osteoblasts on trabecular bone as well as all chondrocyte layers.

\section{Expression of nrf2 in MC3T3-E1 cells}

To examine whether nrf2/Maf signaling molecules are indeed expressed in MC3T3-E1 osteoblastic cells, RT-PCR analysis was conducted. Semi-quantitative RT-PCR revealed that expression of mRNA was drastically increased for osteocalcin during culturing from 14 days with a gradual increase thereafter up to 28 days, while a sustained expression was seen in mRNA expression for both type I collagen and runx2 for a period up to 28 days. Under these conditions, Expression of mRNA for nrf2 was gradually increased 3 to14 days, while no marked alteration was found in Keap1, MafF and MafG mRNA expression at any stages examined.

\section{Effect of nrf2 on osteoblastic differentiation}

To assess whether stable expression of nrf2 could alter the program of osteoblastic differentiation, MC3T3-E1 and ATDC5 cells were stably transfected with pEF containing the full-length coding region of nrf2 (MC3T3-E1-nrf2 and ATDC5-nrf2) or with the empty vector (MC3T3-E1-EV and ATDC5-nrf2). In MC3T3-E1-nrf2 cells, marked decrease was found in alkaline phosphatase activity as well as the expression of type I collagen and osteocalcin when compared with MC3T3-E1-EV cells. In addition, intensity of alizarin red staining and $\mathrm{Ca}^{2+}$ accumulation were also significantly decreased in MC3T3-E1-nrf2 cells.

\section{Effect of nrf2 on runx2-dependent OG2 reporter activity}

An attempt was next made to determine the possible mechanisms of nrf2 on osteoblastic differentiation by determination of reporter activity using mouse osteocalcin promoter, OG2. OG2 reporter activity was significantly enhanced by runx2 expression as previously reported, while no marked increase was found by transfection with nrf2 alone. In contrast, nrf2 significantly impaired the runx2-dependent enhancement of OG2 reporter activity when it was simultaneously transfected with runx2.

\section{Discussion}

The essential importance of the present findings is that the overexpression of nrf2 markedly inhibited osteoblastic differentiation. In addition, nrf2 almost completely inhibited runx2-dependent osteocalcin promoter activity. To our knowledge, this paper deals with the first direct demonstration of functional expression of nrf2 in osteoblasts. Although several previous studies have already demonstrated the functional expression of nrf2 in bone $^{12,13)}$, no direct evidence for a role of it in mechanisms underlying the cellular differentiation in osteoblasts is available in the literature to date.

Nrf2/Maf signaling machineries could be thus a target for the development of a drug useful for the treatment and therapy of a variety of bone diseases relevant to abnormal development and maturation of osteoblasts in human beings.

\section{Acknowledgements}

This work was supported in part by Grants-in-Aid for Scientific Research from the Ministry of Health, Labour and Welfare, Japan.

\section{References}

1. Boyle WJ, Simonet WS and Lacey DL. Osteoclast differentiation and activation. Nature 423:337-342, 2003

2. Harada S and Rodan GA. Control of osteoblast function and regulation of bone mass. Nature 423:349-355, 2003

3. Ducy P, Schinke T and Karsenty G The osteoblast: a sophisticated fibroblast under central surveillance. Science 289:1501-1504, 2000

4. Teitelbaum SL. Bone resorption by osteoclasts. Science 289:1504-1508, 2000

5. Otto F, Thornell AP, Crompton T, Denzel A, Gilmour KC, Rosewell IR, Stamp GW, Beddington RS, Mundlos S, Olsen $\mathrm{BR}$ et al. Cbfa1, a candidate gene for cleidocranial dysplasia syndrome, is essential for osteoblast differentiation and bone development. Cell 89:765-771, 1997

6. Komori T, Yagi H, Nomura S, Yamaguchi A, Sasaki K, Deguchi K, Shimizu Y, Bronson RT, Gao YH, Inada M et al. Targeted disruption of cbfa1 results in a complete lack of bone formation owing to maturational arrest of Osteoblasts. Cell 89:755-764, 1997

7. Ducy P, Zhang R, Geoffroy V, Ridall AL and Karsenty G. Osf2/Cbfa1: a transcriptional activator of osteoblast differentiation. Cell 89:747-754, 1997

8. Takeda S, Bonnamy JP, Owen MJ, Ducy P and Karsenty G. Continuous expression of cbfa1 in nonhypertrophic condrocytes uncovers its ability to induce hypertrophic condrocyte differentiation and partially rescues cbfa1deficient mice. Genes Dev. 15:467-481, 2001

9. Motohashi $\mathrm{H}$ and Yamamoto M. Nrf2-Keap1 defines a physiologically important stress response mechanism. Trends Mol Med 10:549-557, 2004

10.Zhang Y and Gordon GB. A strategy for cancer prevention: stimulation of the Nrf2-ARE signaling pathway. Mol Cancer Ther 3:885-893, 2004

11.Lee JS and Surh YJ. Nrf2 as a novel molecular target for chemoprevention. Cancer Lett 224:171-184, 2005

12.Li B, Wang X, Rasheed N, Hu Y, Boast S, Ishii T, Nakayama K, Nakayama KI and Goff SP. Distinct roles of c-Abl and Atm in oxidative stress response are mediated by protein kinase C delta. Genes Dev 18:1824-1837, 2004

13.Beck GR, Moran E and Knecht N. Inorganic phosphate regulates multiple genes during osteoblast differentiation, including Nrf2. Exp Cell Res 288:288-300, 2003 